Southern Illinois University Edwardsville SPARK

SIUE Faculty Research, Scholarship, and Creative Activity

Fall 10-19-2015

\title{
Odorant receptor-based discovery of natural repellents of human lice
}

\author{
Julien Pelletier \\ University of California-Davis \\ Pingxi $\mathrm{Xu}$ \\ University of California-Davis \\ Kyong-Sup Yoon \\ Southern Illinois University Edwardsville, kyoon@siue.edu \\ John M. Clark \\ University of Massachusetts - Amherst \\ Walter S. Leal \\ University of California-Davis
}

Follow this and additional works at: http://spark.siue.edu/siue_fac

Part of the Behavior and Ethology Commons, Entomology Commons, Other Genetics and Genomics Commons, Other Pharmacology, Toxicology and Environmental Health Commons, and the Parasitology Commons

\section{Recommended Citation}

Pelletier, Julien; Xu, Pingxi; Yoon, Kyong-Sup; Clark, John M.; and Leal, Walter S., "Odorant receptor-based discovery of natural repellents of human lice" (2015). SIUE Faculty Research, Scholarship, and Creative Activity. 15.

http://spark.siue.edu/siue_fac/15

This Article is brought to you for free and open access by SPARK. It has been accepted for inclusion in SIUE Faculty Research, Scholarship, and Creative Activity by an authorized administrator of SPARK. For more information, please contact gpark@siue.edu. 


\section{Cover Page Footnote}

http://dx.doi.org/10.1016/j.ibmb.2015.10.009

(C2015 The Authors. Published by Elsevier Ltd. This is an open access article under the CC BY-NC-ND license (http://creativecommons.org/licenses/by-nc-nd/4.0/) 


\title{
Odorant receptor-based discovery of natural repellents of human lice
}

\author{
Julien Pelletier $^{\mathrm{a}, 1,2}$, Pingxi Xu ${ }^{\mathrm{a}, 1}$, Kyong S. Yoon ${ }^{\mathrm{b}}$, John M. Clark ${ }^{\mathrm{c}}$, Walter S. Leal ${ }^{\mathrm{a},{ }^{*}}$ \\ a Department of Molecular and Cellular Biology, University of California Davis, Davis, CA 95616, USA \\ ${ }^{\mathrm{b}}$ Department of Biological Sciences and Environmental Sciences Program, Southern Illinois University-Edwardsville, Edwardsville, IL 62026, USA \\ c Department of Veterinary and Animal Sciences, University of Massachusetts, Amherst, MA 01003, USA
}

\section{A R T I C L E I N F O}

\section{Article history:}

Received 29 August 2015

Received in revised form

13 October 2015

Accepted 16 October 2015

Available online 19 October 2015

\section{Keywords:}

Lice

Olfaction

Odorant receptors

Olfactory mechanisms

Repellent

Behavior

\begin{abstract}
A B S T R A C T
The body louse, Pediculus humanus humanus, is an obligate blood-feeding ectoparasite and an important insect vector that mediates the transmission of diseases to humans. The analysis of the body louse genome revealed a drastic reduction of the chemosensory gene repertoires when compared to other insects, suggesting specific olfactory adaptations to host specialization and permanent parasitic lifestyle. Here, we present for the first time functional evidence for the role of odorant receptors (ORs) in this insect, with the objective to gain insight into the chemical ecology of this vector. We identified seven putative full-length ORs, in addition to the odorant receptor co-receptor (Orco), and expressed four of them in the Xenopus laevis oocytes system. When screened with a panel of ecologically-relevant odorants, PhumOR2 responded to a narrow set of compounds. At the behavior level, both head and body lice were repelled by the physiologically-active chemicals. This study presents the first evidence of the OR pathway being functional in lice and identifies PhumOR2 as a sensitive receptor of natural repellents that could be used to develop novel efficient molecules to control these insects.
\end{abstract}

() 2015 The Authors. Published by Elsevier Ltd. This is an open access article under the CC BY-NC-ND license (http://creativecommons.org/licenses/by-nc-nd/4.0/).

\section{Introduction}

The human body louse, Pediculus humanus humanus, is an important vector of human pathogens responsible for the transmission of epidemic typhus, trench fever and relapsing fever (Fournier et al., 2002) (Bonilla et al., 2013). The impact of these diseases has been dramatically reduced in recent times, simply because efficient control measures and better hygiene standards in developed countries allowed a near-eradication of the vector (Badiaga et al., 2008) (Brouqui, 2011). However, the reemergence of body lice has occurred in specific areas and populations, maintaining a high epidemiological risk (Raoult and Roux, 1999) (Badiaga et al., 2008) (Brouqui, 2011). Since there are currently no commercial vaccines against louse-borne diseases, control and/or elimination of lice are considered as the best methods available to combat the transmission of these diseases to humans (Bonilla et al., 2013). However, conventional insecticides present several limitations: (1) they do not prevent re-infestation (Mumcuoglu

\footnotetext{
* Corresponding author.

E-mail address: wsleal@ucdavis.edu (W.S. Leal).

1 These authors contributed equally to this study.

2 Current address: School of Life Sciences, Keele University, Keele, ST5 5BG, United Kingdom.
}

et al., 1996); (2) they promote the development of resistance mechanisms in lice (Bonilla et al., 2013); (3) they might cause health problems when applied at high doses to humans (Semmler et al., 2012). In this context, identifying molecules that efficiently repel lice from humans, which protect against re-infestation and have no negative impact on human health, is critical. Several types of repellent products have been considered for louse control, including broad-spectrum synthetics such as N,N-diethyl-3methylbenzamide (DEET) and plant-derived compounds such as essential oils and some of their constituents (e.g. citronellal) (Peock and Maunder, 1993) (Burgess, 1993) (Mumcuoglu et al., 1996) (Mumcuoglu et al., 2004) (Toloza et al., 2006a) (Toloza et al., 2006b) (Toloza et al., 2008) (Canyon and Speare, 2007) (Semmler et al., 2010). Nevertheless, no specific anti-louse repellent has been discovered to date (Semmler et al., 2012) (Burgess et al., 2014).

A main limitation regarding the development of molecules with repellent activity lies in the very limited comprehension of their mode of action at the insect level. Such strategies would likely benefit from a better understanding of the interactions between chemicals and the sensory system of the insect. Most insects rely heavily on chemoreception as it provides a highly valuable link between volatile cues (odorants) from the environment and critical behaviors such as attraction and avoidance (Touhara and Vosshall, 
2009) (Carey and Carlson, 2011) (Leal, 2013). In insects, olfaction takes place in olfactory receptor neurons (ORNs) housed principally in the antennae, the main olfactory organ. At the molecular level, a diverse array of odorant receptors (ORs), ionotropic receptors (IRs), odorant binding proteins (OBPs) and chemosensory proteins (CSPs) have been shown to interact with specific sets of ligands and to play major roles in odorant detection, contributing to the enormous evolutionary success of insects (Benton et al., 2009) (Carey and Carlson, 2011) (Leal, 2013). The publication of the genome sequence of the human body louse showed very limited repertoires of chemosensory genes when compared to other insect species with sequenced genomes, with only ten ORs, twelve IRs, five OBPs, and seven CSPs identified (Kirkness et al., 2010) (Croset et al., 2010). This drastic reduction of the chemoreception machinery is thought to reflect the particular lifestyle of this insect as an obligate ectoparasite which lives in the cloths and feeds solely on the blood of human hosts. This extreme ecological specialization likely goes along with a reduced capacity to locate alternative food sources, prospect for oviposition substrates or detect a variety of natural enemies, but nothing is known about the olfactory adaptations underlying the ecology and behavior of this insect.

To understand the contribution of the louse olfactory system to host specificity and ecological specialization, we took advantage of the genome sequence to identify, clone and functionally characterize OR genes. Odorant receptors represent valuable targets since they ensure the direct interaction with odor ligands, eliciting signal transduction mechanisms that will ultimately lead to specific behaviors (Touhara and Vosshall, 2009) (Carey and Carlson, 2011) (Leal, 2013). In addition, insect ORs have been shown to undergo rapid evolution (Robertson et al., 2003) (Sanchez-Gracia et al., 2009), which is consistent with a role in the adaptation to different ecological environments. A search of the genome data revealed eight full-length putative OR genes, confirming the limited range of this family in this insect. Four ORs were cloned and expressed in the Xenopus laevis oocytes system where they were challenged with a set of ecologically-relevant odorants. Three ORs remained non-responsive but PhumOR2 responded to a narrow set of odorants. Both head and body lice were repelled by the physiologically-active molecules, with two compounds showing high biological activity. This work marks the first step towards a better understanding of chemical communication mechanisms in lice and demonstrates the potential of an odorant receptor as a biological repellent detector towards the development of novel control strategies against this insect vector.

\section{Materials and methods}

\subsection{Identification and cloning of $P$. $h$. humanus odorant receptors}

\subsubsection{Genome search}

Search for ORs in the P. h. humanus genome (Assembly PhumU2, Gene set Phum2.1) was performed by Blast homology search using known Drosophila melanogaster OR sequences as queries. Blast algorithm was used to identify putative ORs in the predicted peptide sequences database in VectorBase. The putative lice OR sequences were screened for the presence of typical OR domains in the NCBI Conserved Domains Database (CDD) and aligned with known insect $\mathrm{OR}$ sequences to assess sequence integrity. The original Vectorbase annotation names for putative $P$. $h$. humanus ORs were used in this study (Table 1).

\subsubsection{Cloning}

Total RNA was extracted from body louse females (wholebody) using Trizol (MRC, Cincinnati, $\mathrm{OH}$ ), following the manufacturer's instructions. First-strand cDNA was synthesized from the RNA template $(5 \mu \mathrm{g})$ using Superscript ${ }^{\mathrm{TM}}$ III reverse transcriptase (Invitrogen, Carlsbad, CA) and oligo(dT) $12-18$ primers in a $20 \mu \mathrm{l}$ reaction. The following gene-specific primers were designed to amplify the coding sequences of seven putative full-length OR genes (Table 1): PHUM213810-F (PhumORco): 5' ATGGGAAAGTACAAACCTCACGGATTGG-3'; PHUM213810-R (PhumORco): TTATTTCAGTTGAACTAAAACCATGAAATA-3'; PHUM225140-F: 5'ATGAAAAATCATATAGATCTTCACATACAT-3'; PHUM225140-R: 5' TCAAGGAATATATTTTTTAGAATTGTTCAG-3'; PHUM430460-F: 5'ATGGAGGAGTTTACCGGATACGAAAAAT-3'; PHUM430460-R: $5^{\prime}-$ TTATTTTTCGTCTCTGATTTGAAGTAAAAA-3'; PHUM318760-F: 5' ATGAGTTTTTTCAATTTGGATTATTTTAAA-3'; PHUM318760-R: $5^{\prime}$ TTAATGTTTTCTCGCCGTTTGATATGCA-3'; PHUM318770-F: $5^{\prime}$ ATGGAAAAAAATTTTGAAAATCACGTTTAT-3'; PHUM318770-R: $5^{\prime}-$ CTAATTGTTATTTTTTTCTCTCATTATTTG-3'; PHUM080360-F: $5^{\prime}-$ ATGAAATCAAATTTTAACGAATTTTTTTTTTC-3'; PHUM080360-R: 5'-TTACTTGATTTCAAATTGTCTTATTAACAT-3'; PHUM600410-F: 5'ATGGAAGAAAATAATAATTTATCTAATTCT-3'; PHUM600410-R: $5^{\prime}$ TTATTTAGATTCCAATTGCCAAAAAAAC-3'. Full-length ORs were amplified by PCR (Pfu Ultra II polymerase, Agilent Technologies, Santa Clara, CA) in $25 \mu$ reactions containing $1 \mu$ l of a whole-body cDNA template and $100 \mathrm{nM}$ of each primer. The following cycling conditions were used: $95^{\circ} \mathrm{C}$ for $1 \mathrm{~min}$ for the initial denaturation step, followed by 40 cycles at $95^{\circ} \mathrm{C}$ for $1 \mathrm{~min}, 54^{\circ} \mathrm{C}$ for $30 \mathrm{~s}, 72^{\circ} \mathrm{C}$ for $1 \mathrm{~min}$ and a final $72^{\circ} \mathrm{C}$ for 5 min elongation step. PCR products were purified from agarose gel (QIAquick Gel Extraction Kit, Qiagen, Valencia, CA) and ligated into blunt-end EcoRV-digested pBlueScript SK+ (T4 DNA ligase, Promega, Madison, WI). Ligation products were used to transform competent cells (One Shot OmniMAX, Invitrogen, Carlsbad, CA), positive clones were grown in LB medium containing ampicillin and plasmids were purified (QIAprep Spin Miniprep Kit, Qiagen, Valencia, CA) and sequenced (Davis Sequencing Inc, Davis, CA). Several independent clones were obtained for 5 putative full-length ORs. Clones for PHUM213810 (PhumOrco), PHUM225140 (PhumOR2) and PHUM600410 (PhumOR7) were identical to database sequences at the amino acid level, whereas clones for PHUM318760 (PhumOR4) and PHUM080360 (PhumOR6) differed slightly from the database versions at the amino acids level. The sequences for PhumOrco, PhumOR2, PhumOR4, PhumOR6 and PhumOR7 were deposited into GenBank under the accession numbers KT369093, KT369094, KT369095, KT369096 and KT369097, respectively.

\subsubsection{Sub-cloning}

Putative full-length ORs were amplified by PCR (Pfu Ultra II polymerase) from pBluescript SK+ plasmid templates using genespecific primers containing restriction enzyme recognition sites, PCR products were digested with appropriate combinations of restriction enzymes (XmaI, BamHI and/or EcoRI, New England Biolabs, Ipswich, MA), purified from agarose gel (QIAquick Gel Extraction Kit) and ligated into pre-digested pGEMHE plasmids (T4 DNA ligase, Promega). Ligation products were used to transform competent cells (One Shot OmniMAX, Invitrogen), positive clones were grown in LB medium containing ampicillin and plasmids were purified and sequenced (Davis Sequencing Inc, Davis, CA).

\subsection{Expression of odorant receptors in X. laevis oocytes}

Using the pGEMHE-PhumOR plasmids as templates, capped cRNAs were synthesized with mMACHINE T7 Kit following the manufacturer's instructions (Xu et al., 2013). Purified OR cRNAs were re-suspended in nuclease-free water at $200 \mathrm{ng} / \mu \mathrm{l}$ and $18.4 \mathrm{nl}$ of cRNAs were microinjected with the same amount of PhumOrco cRNA into stage V or VI X. laevis oocytes (purchased from EcoCyte Bioscience, Austin, TX). Injected oocytes were kept at $18{ }^{\circ} \mathrm{C}$ for $3-7$ 
Table 1

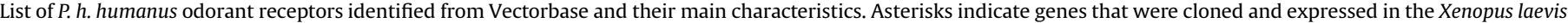
oocytes system.

\begin{tabular}{|c|c|c|c|}
\hline $\begin{array}{l}\text { Vectorbase and NCBI } \\
\text { accession numbers }\end{array}$ & $\begin{array}{l}\text { Vectorbase annotation NCBI conserved } \\
\text { domain database (e-value) }\end{array}$ & Size (aa) & Remarks \\
\hline PHUM213810 & Odorant receptor, putative & \multirow[t]{2}{*}{475} & \multirow[t]{2}{*}{ Full-length Orco* } \\
\hline EEB12924 & 7tm_6 pfam02949 (6.64e-31) & & \\
\hline PHUM318760 & Odorant receptor PhumOR4 & \multirow[t]{2}{*}{409} & \multirow[t]{2}{*}{ Full-length ${ }^{*}$} \\
\hline EEB14695 & 7tm_6 pfam02949 (3.35e-26) & & \\
\hline PHUM318770 & Odorant receptor PhumOR5 & \multirow[t]{2}{*}{402} & \multirow[t]{2}{*}{ Full-length } \\
\hline EEB14696 & 7tm_6 pfam02949 (1.34e-23) & & \\
\hline PHUM600410 & Odorant receptor PhumOR7 & \multirow[t]{2}{*}{411} & \multirow[t]{2}{*}{ Full-length* } \\
\hline EEB20031 & 7tm_6 pfam02949 (4.91e-30) & & \\
\hline PHUM600520 & Predicted protein & \multirow[t]{3}{*}{340 (399) } & Incomplete \\
\hline EEB20032 & 7tm_6 pfam02949 (6.70e-09) & & \multirow[t]{2}{*}{ (reconstructed full-length sequence based on genome sequence) } \\
\hline & 7tm_6 pfam02949 (1.48e-07) & & \\
\hline PHUM600630 & Odorant receptor PhumOR12 & \multirow[t]{2}{*}{189} & \multirow[t]{2}{*}{ Incomplete, lacks $5^{\prime}$ end, putative pseudogene } \\
\hline EEB20033 & 7tm_6 pfam02949 (1.42e-07) & & \\
\hline PHUM225140 & Odorant receptor PhumOR2 & \multirow[t]{2}{*}{406} & \multirow[t]{2}{*}{ Full-length $^{*}$} \\
\hline EEB13133 & 7tm_6 pfam02949 (1.58e-09) & & \\
\hline PHUM080360 & Odorant receptor PhumOR6 & \multirow[t]{2}{*}{419} & \multirow[t]{2}{*}{ Full-length ${ }^{*}$} \\
\hline EEB10972 & 7tm_6 pfam02949 (2.80e-17) & & \\
\hline PHUM430460 & Odorant receptor PhumOR3 & \multirow[t]{2}{*}{434} & \multirow[t]{2}{*}{ Full-length } \\
\hline EEB16632 & 7tm_6 pfam02949 (2.92e-13) & & \\
\hline
\end{tabular}

days in modified Barth's solution [ $\mathrm{NaCl} 88 \mathrm{mM}, \mathrm{KCl} 1 \mathrm{mM}, \mathrm{NaHCO}_{3}$ $2.4 \mathrm{mM}, \mathrm{MgSO}_{4} 0.82 \mathrm{mM}, \mathrm{Ca}\left(\mathrm{NO}_{3}\right)_{2} 0.33 \mathrm{mM}, \mathrm{CaCl}_{2} 0.41 \mathrm{mM}$, HEPES $10 \mathrm{mM}, \mathrm{pH} 7.4$ ] supplemented with $10 \mu \mathrm{g} / \mathrm{ml}$ of gentamicin, $10 \mu \mathrm{g} /$ $\mathrm{ml}$ of streptomycin and $1.8 \mathrm{mM}$ sodium pyruvate.

\subsection{Odor panel and electrophysiological recordings}

Two-electrode voltage-clamp technique (TEVC) was employed to observe odorant-induced currents at holding potential of $-80 \mathrm{mV}$. Signals were amplified with an OC-725C amplifier (Warner Instruments, Hamden, CT), low-pass filtered at $50 \mathrm{~Hz}$ and digitized at $1 \mathrm{kHz}$. Data acquisition was carried out with Digidata 1440 A. Data analysis was processed by software pCLAMP 10 (Molecular Devices, LLC, Sunnyvale, CA) and GraphPad Prism 6 (La Jolla, CA). The following panel of compounds was used to deorphanize ORs: 1-butanol, 1-pentanol, 1-hexanol, 1-heptanol, 1octanol, 1-nonanol, 2,3-butanediol, 2-butoxyethanol, 3-methyl-1butanol, trans-2-hexen-1-ol, cis-2-hexen-1-ol, 1-hexene-3-ol, 1heptene-3-ol, 3-octanol, 1-octen-3-ol, 1-octyn-3-ol, 3-octyn-1-ol, trans-2-nonen-1-ol, cis-2-nonen-1-ol, 4-methylcyclohexanol, pmenthane-3,8-diol, methyl acetate, ethyl acetate, propyl acetate, butyl acetate, pentylacetate, hexyl acetate, nonyl acetate, decyl acetate, methyl propionate, ethyl propionate, methyl butyrate, ethyl butanoate, methyl hexanoate, $(E)$-2-hexenyl acetate, ethyl lactate, methyl salicylate, 1-octen-3-yl acetate, isopentyl acetate, m-tolyl acetate, ethyl phenylacetate, geranyl acetate, propanal, butanal, pentanal, hexanal, heptanal, octanal, nonanal, decanal, undecanal, 1-dodecanal, (E)-2-hexenal, (E)-2-nonenal, phenylacetaldehyde, furfural, 2-butanone, 2-heptanone, geranyl acetone, 6-methyl-5-hepten-2-one, 5-methyl-2-hexanone, 2,3butanedione, 3-hydroxy-2-butanone, 2-undecanone, 2tridecanone, 2-nonanone, fenchone, cyclohexanone, acetophenone, lactic acid, dodecanoic acid (lauric acid), ethanoic acid, propanoic acid, butanoic acid, isobutyric acid, 2-oxobutyric acid, pentanoic acid, 2-oxovaleric acid, isovaleric acid, hexanoic acid, $(E)$ 2-hexanoic acid, 5-hexanoic acid, (E)-3-hexenoic acid, heptanoic acid, octanoic acid, nonanoic acid, decanoic acid, triethylamine, ntridecanoic acid, linoleic acid, ammonia, propylamine, butylamine, pentylamine, hexylamine, heptylamine, octylamine, 1,4diaminobutane, 1,5-diaminopentane, benzaldehyde, phenol, 2methylphenol, 3-methylphenol, 4-methylphenol, 4-ethylphenol, 3,5-dimethylphenol, 2,3-dimethylphenol, guaiacol, 2-methoxy-4- propylphenol, 2-phenoxyethanol, 1,2-dimethoxybenzene, benzyl alcohol, 2-phenylethanol, 1-phenylethanol, phenylether, isoprene, limonene, $\alpha$-humulene, linalool oxide, geraniol, nerol, thujone, linalool, eucalyptol, citral, eugenol, $\alpha$-pinene, ocimene, $( \pm)$-citronellal, indole, 1-methylindole, 2-methylindole, 3-methylindole, 4methylindole, 5-methylindole, 6-methylindole, 7-methylindole, $\gamma$ valerolactone, $\gamma$-hexalactone, $\gamma$-octalactone, $\gamma$-decalactone, 2 acetylthiophene, dimethyl phthalate, isovaleraldehyde, ethyl 3hydroxyhexanoate and $\mathrm{N}, \mathrm{N}$-diethyl-3-methylbenzamide (DEET). All compounds were 95\% pure or higher (except for trimethylamine, which was used as a $45 \%$ water solution). Chemicals were prepared in DMSO as $1 \mathrm{M}$ stock solutions and subsequently diluted in oocyte Ringer buffer at $10^{-3} \mathrm{M}$ for physiology screening experiments. All chemicals were purchased from Sigma-Aldrich (St. Louis, MO), except for p-menthane-3,8-diol, which was obtained from Bedoukian Research Inc. (Danbury, CT).

\subsection{Behavior experiments}

\subsubsection{Chemicals}

4-methylcyclohexanol (98\% pure), 2,3-dimethylphenol (99\% pure) and 1-phenylethanol (98\% pure) were purchased from Sigma-Aldrich (St. Louis, MO).

\subsubsection{Insect samples}

Permethrin- and DDT-resistant human head lice (SF-HL) were collected from infested children in Plantation and Homestead, FL. Insecticide-susceptible human body lice (USDA-BL) were provided by Dr. Kosta Mumcuoglu (Hebrew University, Jerusalem, Israel) who originally obtained his body louse colony from USDA laboratory (Gainesville, FL). Human head and body lice (SF-HL and USDABL) were maintained at the University of Massachusetts at Amherst, MA as described previously (Yoon et al., 2006) (Johnston et al., 2007). Adult female lice (10/trial) were randomly selected from the colony and used in all experiments.

\subsubsection{Test arena}

All behavioral bioassays were performed inside a fabricated glass test arena. The test arena $(75 \times 25 \times 25 \mathrm{~mm})$ was constructed from standard glass microscope slides and held together with epoxy glue. Distilled deionized water $\left(\mathrm{ddH}_{2} \mathrm{O}\right)$, ethanol and hexane were consecutively used to clean the test arena prior to each 
experiment. The cleaned test arena was placed in a $950 \mathrm{ml}$ glass container with a plastic cover (Pyrex Round Storage, Corning, NY) and stored at room temperature until used. A single layer of a MIRASORB ${ }^{\circledR}$ gauze patch (Johnson \& Johnson, New Brunswick, NJ) was cut into two rectangular patches of equal dimensions $(37.5 \times 25 \mathrm{~mm}$ ). Each patch was cut to exactly fit the bottom of the glass arena. This configuration allowed lice to move on the top surface of the patches freely without falling off or leaving the test arena.

\subsubsection{Behavioral assays}

For repellency bioassays, a gauze patch $(37.5 \times 25 \mathrm{~mm})$ on one half of the arena was treated with a test compound (4methylcyclohexanol, 2,3-dimethylphenol or 1-phenylethanol) dissolved in $250 \mu$ l ethanol ( $1 \% \mathrm{w} / \mathrm{v}$ and additionally $0.01 \% \mathrm{w} / \mathrm{v}$ for $2,3-$ dimethylphenol), and a control patch on the other half of the arena was treated with ethanol only $(250 \mu \mathrm{l})$. For control experiments, a gauze patch $(37.5 \times 25 \mathrm{~mm})$, which was fitted to one half of the bottom surface area of the test arena, was treated with ethanol only and the other identical patch on the other half of the arena received no treatment (no-treatment patch). For treatment experiments, one patch received ethanol only and the other patch received a test compound in ethanol. All patches were air dried in a dark fume hood for $1 \mathrm{~h}$. Adult female lice (10/trial) were simultaneously placed on the boundary between the two patches and the arena was placed on a laboratory bench at room temperature for $30 \mathrm{~min}$. Following this interval, the number of lice found on each patch was recorded. Repellency percentage values were calculated using the following equation:

$\%$ repellency $=100-[(\mathrm{nt} / \mathrm{Nt}) /(\mathrm{nc} / \mathrm{Nc}) \times 100]$, where $\mathrm{Nc}$ indicates the total number of female in the control arena, nc indicates the total number of females on the ethanol-treated patch in the control arena, Nt indicates the total number of female in the treatment arena, nt indicates the total number of females on the test compound-treated patch in the treatment arena.

\section{Results and discussion}

\subsection{Identification of the odorant receptor repertoire in $P . h$. humanus}

Analysis of the human body louse genome (Assembly PhumU2, Gene set Phum2.1) allowed the identification of nine putative OR genes by Blast search (Table 1). A gene annotated as a putative OR in Vectorbase (PHUM327990) was not included in our gene set since it did not display any structural features characteristic of the insect OR family. Among putative ORs, one gene (PHUM213810) encodes a full-length OR co-receptor (Orco) (Larsson et al., 2004), six genes (PHUM318760, PHUM318770, PHUM600410, PHUM225140, PHUM080360 and PHUM430460) likely encode full-length ORs and two genes (PHUM600520 and PHUM600630) appeared to encode only partial ORs, based on multiple alignments with other insect ORs. Among the partial sequences, we were able to reconstruct a putative full-length sequence for PHUM600520 using the genome sequence, but failed to reconstruct PHUM600630, suggesting the latter might be a true pseudogene. The last exon of the pseudogene PHUM600630 displays very high identity with PHUM600520, but only moderate identity for the rest of its sequence, indicating that this pseudogene might be a mix of at least two partial OR genes, probably resulting from annotation errors. Regarding the evolution of this gene family, we identified two genomic clusters encompassing two (PHUM318760 and PHUM318770) and three genes (PHUM600410, PHUM600520 and the putative pseudogene PHUM600630), suggesting that gene duplications contributed to shape the repertoire of ORs in this species. Overall, $P$. $h$. humanus
ORs display very little similarity between them, even across putative paralogs. The highest conservation occurs between PHUM080360 and PHUM600410 (around 49\% identity at the amino acids level), two genes which are located in different areas of the genome (Table 2). When compared with ORs from other insects, only PhumOrco displayed significant identity (around 60\% at the amino acids level) with orthologous proteins from other species (Table 2). A high level of sequence divergence within and across species represents a traditional trademark of the OR family (Robertson et al., 2003) (Sanchez-Gracia et al., 2009). Among putative full-length ORs, PhumOrco (PHUM213810), PhumOR2 (PHUM225140), PhumOR4 (PHUM318760), PhumOR6 (PHUM080360) and PhumOR7 (PHUM600410) were successfully cloned, whereas PhumOR3 (PHUM430460) and PhumOR5 (PHUM318770) could not be amplified from a body louse wholebody cDNA template (Table 1 ). To further investigate the molecular mechanisms of odorant reception in the body louse, we used this set of four ORs for functional characterization in the X. laevis oocyte heterologous expression system.

\subsection{An odorant receptor sensitive to a subset of odorants}

Nothing was known about chemical communication and olfactory mechanisms in $P$. $h$. humanus at the beginning of this study. Earlier morphological studies showed the presence of sensory structures resembling olfactory sensilla on the tip of the antennae (described as peg organs and pore organs) of the human body louse (Slifer and Sekhon, 1980) (Steinbrecht, 1994), but their specific involvement in olfaction remained unclear.

For the deorphanization of a subset of louse ORs, we selected a set of 150 compounds that represent common volatiles in nature and other structure-related chemicals. Odorant receptors were coexpressed with Orco in the $X$. laevis oocytes system, a robust tool that has been used to decipher the function of multiple insect ORs, including ORs from the malaria mosquito and other vector of diseases (Bohbot and Dickens, 2009) (Pelletier et al., 2010) (Wang et al., 2010). When screened with the panel of odorants, PhumOR4, PhumOR6 and PhumOR7 did not respond to any test compounds. Different hypotheses could explain this lack of receptivity: (1) these ORs could be narrowly tuned to one or a few compounds that were not represented in the test panel, which is consistent with the lice extreme ecological specialization; (2) the clones that were used in the heterologous system could be non-responsive alleles, even if this is not likely considering that we sequenced multiple clones for each transcript. In addition, most cloned sequences were very similar or strictly identical to the reference sequences in databases; (3) the clones could be refractory for functional expression in the Xenopus oocytes heterologous system, possibly because the injected cRNAs did not translate properly or did not produce functional receptor proteins.

Contrarily to the above ORs, PhumOR2 responded to several odorants from the panel, providing clear evidence that the OR pathway is functional in lice (Fig. 1). The most active ligands were found within phenols, benzene alcohols, indoles and alcohols, indicating that the receptor is not tuned to a single chemical class. Overall, the best ligands among these classes were 2,3dimethylphenol, 1-phenylethanol and 4-methylcyclohexanol, all of which elicited much stronger responses than other test compounds, suggesting that PhumOR2 is highly selective. This discovery prompted us to test the activity of these odorants at different doses, revealing that PhumOR2 responded to these chemicals in a dose-dependent manner, showing the highest sensitivity to 2,3dimethylphenol (EC50: $\left.1.03 \times 10^{-4} \mathrm{M}\right)$, 4-methylcyclohexanol (EC50: $\left.1.2 \times 10^{-4} \mathrm{M}\right)$, then 1-phenylethanol (EC50: $1.3 \times 10^{-3} \mathrm{M}$ ) (Fig. 2). Overall, the response profile indicates that PhumOR2 is 
Table 2

List of $P$. h. humanus odorant receptors and their closest homologs in lice and other insects.

\begin{tabular}{|c|c|c|}
\hline Lice odorant receptor & $\begin{array}{l}\text { Most related lice homolog } \\
\text { (amino acid identity \%) (query coverage \%) }\end{array}$ & Most related insect homolog (amino acid identity \%) (query coverage \%) \\
\hline PHUM213810 & \multirow[t]{2}{*}{ PHUM600410 (PhumOR7) (24\%) (33\%) } & Tribolium castaneum Orco (XP_008194693) (62\%) (100\%) \\
\hline PhumOrco & & Tenebrio molitor Orco (AJO62219) (62\%) (100\%) \\
\hline PHUM318760 & \multirow[t]{2}{*}{ PHUM600410 (PhumOR7) (22\%) (95\%) } & \multirow[t]{2}{*}{ Wasmannia auropunctata OR2 (XP_011691922) (22\%) (95\%) } \\
\hline PhumOR4 & & \\
\hline PHUM318770 & \multirow{2}{*}{ PHUM600410 (PhumOR7) (29\%) (97\%) } & \multirow[t]{2}{*}{ Helicoverpa armigera OR (AIG51906) (23\%) (93\%) } \\
\hline PhumOR5 & & \\
\hline PHUM600410 & \multirow[t]{2}{*}{ PHUM080360 (PhumOR6) (49\%) (97\%) } & \multirow[t]{2}{*}{ Ostrinia furnacalis OR24 (BAR43466) (25\%) (60\%) } \\
\hline PhumOR7 & & \\
\hline PHUM600520 (reconstructed) & PHUM600410 (PhumOR7) (26\%) (94\%) & Vollenhovia emeryi OR22c-like (XP_011862397) (29\%) (46\%) \\
\hline PHUM600630 & \multirow[t]{2}{*}{ NA: likely a mix of different partial OR sequences } & \multirow[t]{2}{*}{ NA: likely a mix of different partial OR sequences } \\
\hline PhumOR12 (pseudogene) & & \\
\hline PHUM225140 & \multirow[t]{2}{*}{ PHUM213810 (PhumOrco) (20\%) (39\%) } & Helicoverpa assulta OR43, partial sequence (AJD81577) \\
\hline PhumOR2 & & $(23 \%)(53 \%)$ \\
\hline PHUM080360 & \multirow[t]{2}{*}{ PHUM600410 (PhumOR7) (49\%) (97\%) } & \multirow[t]{2}{*}{ Danaus plexippus OR60 (EHJ76513) (24\%) (98\%) } \\
\hline PhumOR6 & & \\
\hline PHUM430460 & \multirow[t]{2}{*}{ PHUM600410 (PhumOR7) (23\%) (74\%) } & \multirow[t]{2}{*}{ Fopius arisanus OR22c-like (XP_011301209) (22\%) (91\%) } \\
\hline PhumOR3 & & \\
\hline
\end{tabular}

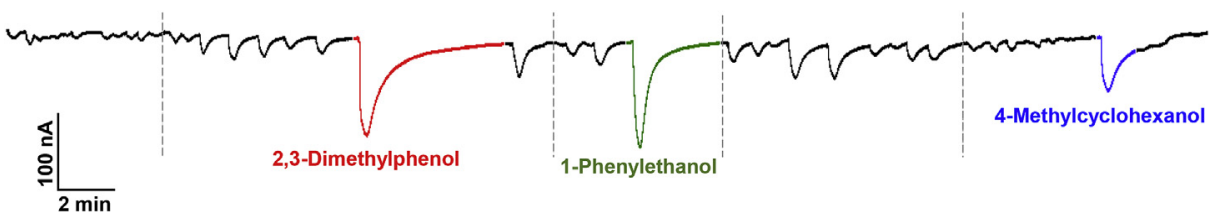

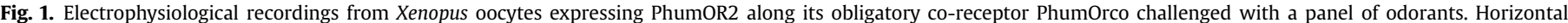

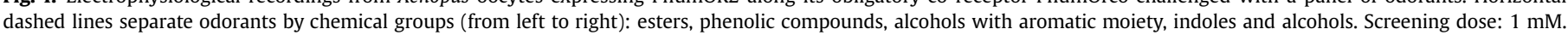

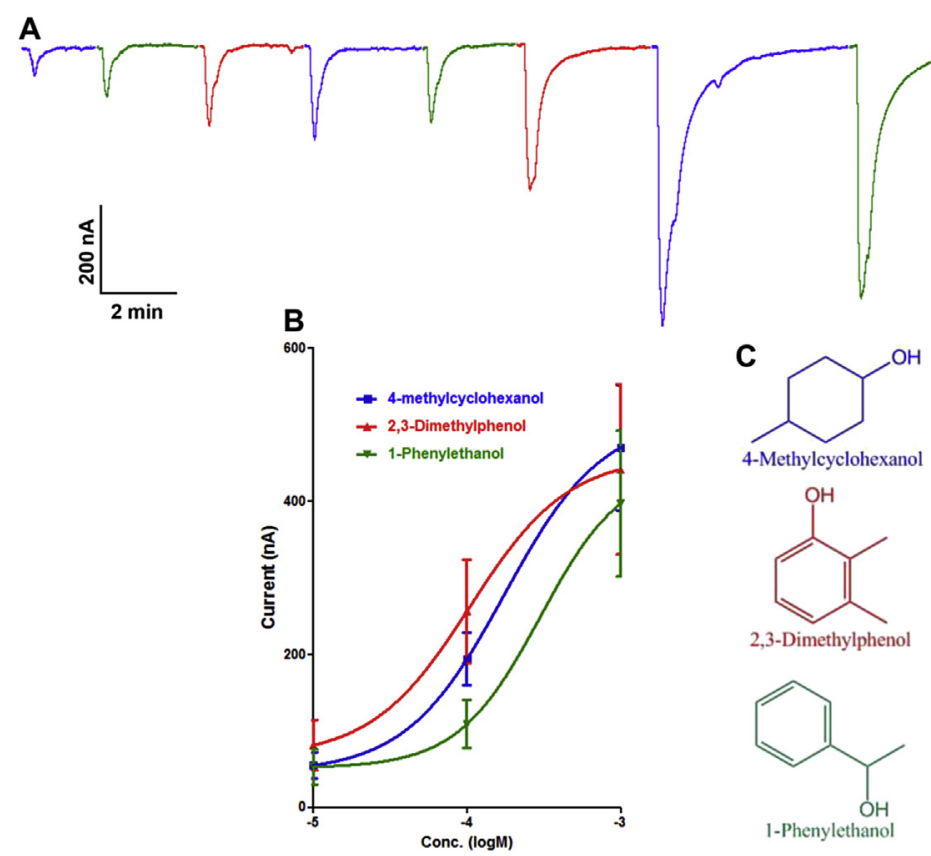

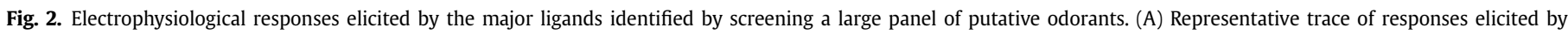

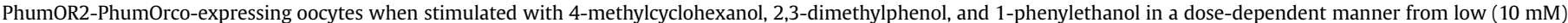
to high dose $(1 \mathrm{mM})$. (B) Dose-dependent relationships $(N=4)$. (C) Chemical structures of the major ligands.

involved in the reception of a narrow set of odorants. In the mosquito Anopheles gambiae, narrowly tuned ORs were found to be strongly activated by odorants with high ecological significance (Wang et al., 2010) (Carey et al., 2010), suggesting that PhumOR2 ensures the reception of odorants with high biological relevance. To understand the significance of these physiologically-active ligands in lice, behavioral assays were carried out.

\subsection{Lice avoidance to physiologically-active chemicals}

A glass test arena was used to measure the behavioral response of body and head lice towards the three most physiologically-active compounds identified by using PhumOR2 as a chemical detector (Fig. 3). In all cases except where indicated, a $250 \mu \mathrm{l}$ aliquot of a $1 \%$ test solution was applied to the gauze test patch $\left(\sim 3.7 \mu \mathrm{mol} / \mathrm{cm}^{2}\right)$. 
2,3-dimethylphenol was also applied as a $0.01 \% \mathrm{w} / \mathrm{v}$ solution $\left(\sim 37 \mathrm{nmol} / \mathrm{cm}^{2}\right)$. All three odorants elicited clear avoidance behavior with similar effects observed for both species (Table 3 ). The strongest mean percent repellency for body and head lice was observed for 2,3-dimethylphenol (96.9\% and $80.8 \%$, respectively), followed by 4 -methylcyclohexanol ( $71.4 \%$ and $78.0 \%$, respectively) and then 1-phenylethanol (42.9\% and 47.5\%, respectively). Significant repellency was also observed for 2,3-dimethylphenol at a concentration of $0.01 \%$ applied to the test patch. These experiments indicate that both body and head lice are able to perceive a common set of semiochemicals that are mediating similar behavior, suggesting that chemical communication mechanisms are highly conserved in both louse ecotypes. Interestingly, the intensity of the behavioral responses in lice (Table 3) mimicked the intensity of the physiological responses observed when these compounds were presented at low doses to the oocytes-expressing PhumOR2 (Fig. 2), suggesting that receptor sensitivity is directly correlated with biological activity.

\subsection{Ecological implications regarding the role of odorant receptors} in lice

Analysis of the human body louse genome reported very limited repertoires of chemosensory receptors with only 8 putative fulllength ORs (Table 1) (the genome paper reported a total of 10 ORs), 12 IRs (including 10 putative antennal IRs) and no carbon dioxide detection pathway (Kirkness et al., 2010) (Croset et al., 2010). Thus, it is likely that olfactory-driven behaviors in lice are mediated by a limited number of ORNs expressing either ORs or IRs. The lack of response of PhumOR4, PhumOR6 and PhumOR7 towards the odorant panel suggests that part of the olfactory system may be tuned to intraspecific recognition cues, including pheromones, other body secretions or microbiota-derived odors, rather than the detection of abundant environmental volatiles, even if we cannot rule out that these ORs may be non-functional in the

\section{(A) Control arena}

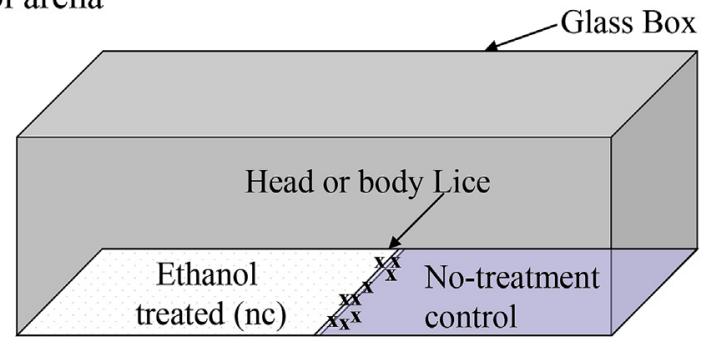

(B) Treatment arena

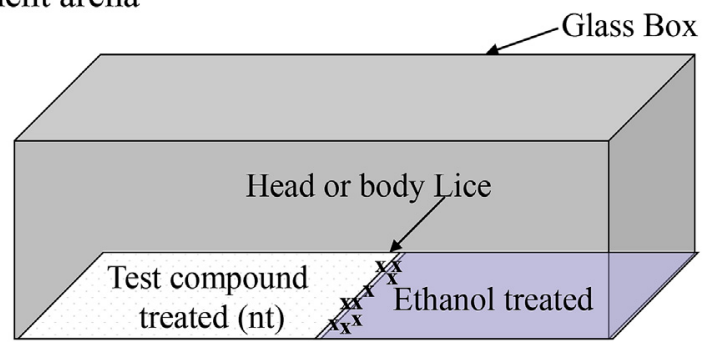

Fig. 3. Behavior bioassay test arena constructed on an open-top glass box $(7.5 \times 7.5 \times 2.5 \mathrm{~cm})$. (A) Control arena prepared with the ethanol $(250 \mu \mathrm{l})$ patch and the no-treatment patch. (B) Treatment arena prepared with the test compound-treated patch and ethanol-treated patch.

\section{Table 3}

Comparative percent repellency (\%) of either female body (USDA) or female head lice (SF-HL) to 2,3-dimethylphenol, 4-methylcyclohexanol, or 1-phenylethanol-treated patches used in the treatment arena. In all cases except where indicated, a $250 \mu \mathrm{l}$ aliquot of a $1 \%$ test solution was applied to the gauze test patch $\left(\sim 3.7 \mu \mathrm{mol} / \mathrm{cm}^{2}\right) .2,3$ dimethylphenol was also applied a $250 \mu$ l aliquot of a $0.01 \%$ test solution $(\sim 37 \mathrm{nmol} /$ $\mathrm{cm}^{2}$ ). Nc indicates the total number of female in the control arena; nc indicates the total number of females on the ethanol-treated patch in the control arena; Nt indicates the total number of female in the treatment arena; nt indicates the total number of females on the test compound-treated patch in the treatment arena. Asterisks indicate repellency percentages that were significantly different from control (Fisher's exact test, $p<0.05$ ).

\begin{tabular}{llll}
\hline Treatment, body lice & $\mathrm{Nc}$ or $\mathrm{Nt}$ & $\mathrm{nc}$ or $\mathrm{nt}$ & Repellency (\%) \\
\hline Control & $\mathrm{Nc}=60$ & $\mathrm{nc}=28$ & - \\
2,3-dimethylphenol & $\mathrm{Nt}=70$ & $\mathrm{nt}=1$ & $96.9^{*}$ \\
4-methylcyclohexanol & $\mathrm{Nt}=60$ & $\mathrm{nt}=8$ & $71.4^{*}$ \\
1-phenylethanol & $\mathrm{Nt}=60$ & $\mathrm{nt}=16$ & $42.9^{*}$ \\
Treatment, head lice & $\mathrm{Nc}$ or Nt & $\mathrm{nc}$ or $\mathrm{nt}$ & Repellency (\%) \\
Control & $\mathrm{Nc}=50$ & $\mathrm{nc}=26$ & - \\
2,3-dimethylphenol & $\mathrm{Nt}=50$ & $\mathrm{nt}=5$ & $80.8^{*}$ \\
0.01\% & $\mathrm{Nt}=30$ & $\mathrm{nt}=7$ & $55.1^{*}$ \\
4-methylcyclohexanol & $\mathrm{Nt}=70$ & $\mathrm{nt}=8$ & $78.0^{*}$ \\
1-phenylethanol & $\mathrm{Nt}=70$ & $\mathrm{nt}=19$ & $47.8^{*}$ \\
\hline
\end{tabular}

Xenopus oocytes system. This finding may be consistent with $P$. $h$. humanus particular lifestyle as a monophagous parasite living in cloths and feeding solely on human hosts. It was shown previously that human body lice were attracted to a filter paper impregnated with an extract of feces (Mumcuoglu et al., 1986). Among excretory products, ammonium bicarbonate was shown to provoke lice aggregation, but the observed effect was less than the effect observed with the whole feces extract, suggesting that additional components are involved in the body louse aggregation behavior. Insects with their antennae removed were not attracted at all, suggesting that olfactory cues present in the feces and received by the antennae are involved in the aggregation behavior. In this context, conventional chemical ecology approaches would be perfectly adapted to identify such ligands from natural substrates (e.g. feces, whole-insect, human skin or sweat). The identification of PhumOR2 as a repellent-sensitive receptor indicates that lice antennae are equipped with a natural avoidance pathway. It was shown previously that individual constituents of essential oils such as citronellal and geraniol were efficient repellents for the human body louse (Mumcuoglu et al., 1996), but those compounds did not elicit any response from test ORs, suggesting that at least an additional ORN expressing another chemoreceptor might be involved in avoidance behaviors. DEET was also shown as an active repellent of the body louse (Mumcuoglu et al., 1996) and did not elicit any response in our receptor screening experiments, but nothing is known regarding the mode of action of this broad-spectrum insect repellent in lice.

\subsection{PhumOR2 as a biological detector of lice repellents}

The idea that natural compounds possess repellent properties against lice is not novel (Burgess, 1993; Burgess et al., 2014). Since then, a wide range of molecules with repellent activities, including natural substances derived from plants as well as synthetics have been developed (Peock and Maunder, 1993) (Burgess, 1993) (Mumcuoglu et al., 1996) (Mumcuoglu et al., 2004) (Toloza et al., 2006a) (Toloza et al., 2006b) (Toloza et al., 2008) (Canyon and Speare, 2007) (Semmler et al., 2010), but nothing was known regarding their mode of action at the insect level. The results of our study indicate that PhumOR2 is linked to avoidance behavior by mediating the reception of natural repellents. Both head and body lice were affected by the biologically-active compounds in our behavior bioassay, which is not surprising considering that both 
ecotypes are very similar at the genetic level and are not considered as different species by most experts (Light et al., 2008) (Veracx and Raoult, 2012) (Bonilla et al., 2013). This discovery paves the way towards the development of novel, cheaper, safer, and more specific molecules with repellent activity to control lice and reduce the transmission of louse-borne diseases. Future experiments should consider exploiting PhumOR2 as a molecular target in large-scale screening bioassays to identify such molecules. Since constituents of essential oils such as citronellal and geraniol that were shown to possess repellent activity against lice (Mumcuoglu et al., 1996) did not activate PhumOR2 in our experiments, we hypothesize that at least another chemoreceptor expressed in another ORN could be involved in avoidance behavior in lice. In that context, future control strategies could exploit a combination of compounds that would activate a couple of independent avoidance-mediating ORNs and possibly generate high repellency effects.

\section{Acknowledgments}

Research reported in this publication was supported by the National institute of Allergy and Infectious Diseases of the National Institutes of Health under Award Number R01AI095514. The content is solely the responsibility of the authors and does not necessarily represent the official views of the National Institutes of Health.

\section{References}

Badiaga, S., Raoult, D., Brouqui, P., 2008. Preventing and controlling emerging and reemerging transmissible diseases in the homeless. Emerg. Infect. Dis. 14, 1353-1359.

Benton, R., Vannice, K.S., Gomez-Diaz, C., Vosshall, L.B., 2009. Variant ionotropic glutamate receptors as chemosensory receptors in Drosophila. Cell 136, 149-162.

Bohbot, J.D., Dickens, J.C., 2009. Characterization of an enantioselective odorant receptor in the yellow fever mosquito Aedes aegypti. PLoS One 4, e7032.

Bonilla, D.L., Durden, L.A., Eremeeva, M.E., Dasch, G.A., 2013. The biology and taxonomy of head and body lice-implications for louse-borne disease prevention. PLoS Pathog. 9, e1003724.

Brouqui, P., 2011. Arthropod-borne diseases associated with political and social disorder. Annu. Rev. Entomol. 56, 357-374.

Burgess, I., 1993. New head louse repellent. Br. J. Dermatol. 128, 357-358.

Burgess, I.F., Brown, C.M., Burgess, N.A., Kaufman, J., 2014. Can head louse repellents really work? Field studies of piperonal 2\% spray. Peerj 2, e351.

Canyon, D.V., Speare, R., 2007. A comparison of botanical and synthetic substances commonly used to prevent head lice (Pediculus humanus var. capitis) infestation. Int. J. Dermatol. 46, 422-426.

Carey, A.F., Carlson, J.R., 2011. Insect olfaction from model systems to disease control. Proc. Natl. Acad. Sci. U. S. A. 108, 12987-12995.

Carey, A.F., Wang, G., Su, C.Y., Zwiebel, L.J., Carlson, J.R., 2010. Odorant reception in the malaria mosquito Anopheles gambiae. Nature 464, 66-71.

Croset, V., Rytz, R., Cummins, S.F., Budd, A., Brawand, D., Kaessmann, H., Gibson, T.J., Benton, R., 2010. Ancient protostome origin of chemosensory ionotropic glutamate receptors and the evolution of insect taste and olfaction. PLoS Genet 6. e1001064.

Fournier, P.E., Ndihokubwayo, J.B., Guidran, J., Kelly, P.J., Raoult, D., 2002. Human pathogens in body and head lice. Emerg. Infect. Dis. 8, 1515-1518.

Johnston, J.S., Yoon, K.S., Strycharz, J.P., Pittendrigh, B.R., Clark, J.M., 2007. Body lice and head lice (Anoplura: Pediculidae) have the smallest genomes of any hemimetabolous insect reported to date. J. Med. Entomol. 44, 1009-1012.

Kirkness, E.F., Haas, B.J., Sun, W., Braig, H.R., Perotti, M.A., Clark, J.M., Lee, S.H., Robertson, H.M., Kennedy, R.C. Elhaik, E. Gerlach, D., Kriventseva, E.V. Elsik, C.G., Graur, D., Hill, C.A., Veenstra, J.A., Walenz, B., Tubio, J.M., Ribeiro, J.M., Rozas, J., Johnston, J.S., Reese, J.T., Popadic, A., Tojo, M., Raoult, D., Reed, D.L., Tomoyasu, Y., Kraus, E., Mittapalli, O., Margam, V.M., Li, H.M., Meyer, J.M. Johnson, R.M., Romero-Severson, J., Vanzee, J.P., Alvarez-Ponce, D., Vieira, F.G., Aguade, M., Guirao-Rico, S., Anzola, J.M., Yoon, K.S., Strycharz, J.P., Unger, M.F.,
Christley, S., Lobo, N.F., Seufferheld, M.J., Wang, N., Dasch, G.A., Struchiner, C.J., Madey, G., Hannick, L.I., Bidwell, S., Joardar, V., Caler, E., Shao, R., Barker, S.C., Cameron, S., Bruggner, R.V., Regier, A., Johnson, J., Viswanathan, L., Utterback, T.R., Sutton, G.G., Lawson, D., Waterhouse, R.M., Venter, J.C., Strausberg, R.L., Berenbaum, M.R., Collins, F.H., Zdobnov, E.M., Pittendrigh, B.R., 2010. Genome sequences of the human body louse and its primary endosymbiont provide insights into the permanent parasitic lifestyle. Proc. Natl. Acad. Sci. U. S. A. 107, 12168-12173.

Leal, W.S., 2013. Odorant reception in insects: roles of receptors, binding proteins, and degrading enzymes. Annu. Rev. Entomol. 58, 373-391.

Light, J.E., Toups, M.A., Reed, D.L., 2008. What's in a name: the taxonomic status of human head and body lice. Mol. Phylogenet. Evol. 47, 1203-1216.

Larsson, M.C., Domingos, A.I., Jones, W.D., Chiappe, M.E., Amrein, H., Vosshall, L.B., 2004. Or83b encodes a broadly expressed odorant receptor essential for Drosophila olfaction. Neuron 43 (5), 703-714.

Mumcuoglu, K.Y., Galun, R., Bach, U., Miller, J., Magdassi, S., 1996. Repellency of essential oils and their components to the human body louse, Pediculus humanus humanus. Entomol. Exp. Appl. 78, 309-314.

Mumcuoglu, K.Y., Magdassi, S., Miller, J., Ben-Ishai, F., Zentner, G., Helbin, V., Friger, M., Kahana, F., Ingber, A., 2004. Repellency of citronella for head lice: double-blind randomized trial of efficacy and safety. Isr. Med. Assoc. J. 6, 756-759.

Mumcuoglu, Y., Galun, R., Ikan, R., 1986. The aggregation response of human-body louse (Pediculus-Humanus) (Insecta, Anoplura) to its excretory products. Insect Sci. Appl. 7, 629-632.

Pelletier, J., Hughes, D.T., Luetje, C.W., Leal, W.S., 2010. An odorant receptor from the southern house mosquito Culex pipiens quinquefasciatus sensitive to oviposition attractants. PLoS One 5, e10090.

Peock, S., Maunder, J.W., 1993. Arena tests with piperonal, a new louse repellent. J. R. Soc. Health 113, 292-294.

Raoult, D., Roux, V., 1999. The body louse as a vector of reemerging human diseases. Clin. Infect. Dis. 29, 888-911.

Robertson, H.M., Warr, C.G., Carlson, J.R., 2003. Molecular evolution of the insect chemoreceptor gene superfamily in Drosophila melanogaster. Proc. Natl. Acad. Sci. U. S. A. 100 (Suppl. 2), 14537-14542.

Sanchez-Gracia, A., Vieira, F.G., Rozas, J., 2009. Molecular evolution of the major chemosensory gene families in insects. Hered. (Edinb) 103, 208-216.

Semmler, M., Abdel-Ghaffar, F., Al-Quraishy, S., Al-Rasheid, K.A.S., Mehlhorn, H., 2012. Why is it crucial to test anti-lice repellents? Parasitol. Res. 110, $273-276$.

Semmler, M., Abdel-Ghaffar, F., Al-Rasheid, K., Klimpel, S., Mehlhorn, H., 2010. Repellency against head lice (Pediculus humanus capitis). Parasitol. Res. 106, 729-731.

Slifer, E.H., Sekhon, S.S., 1980. Sense-organs on the antennal flagellum of the human louse, Pediculus humanus (Anoplura). J. Morphol. 164, 161-166.

Steinbrecht, R.A., 1994. The tuft organs of the human body louse, Pediculus humanus corporis-cryofixation study of a thermo-/hygrosensitive sensillum. Tissue Cell 26, 259-275.

Toloza, A.C., Lucia, A., Zerba, E., Masuh, H., Picollo, M.I., 2008. Interspecific hybridization of Eucalyptus as a potential tool to improve the bioactivity of essential oils against permethrin-resistant head lice from Argentina. Bioresour. Technol. 99, 7341-7347.

Toloza, A.C., Zygadlo, J., Cueto, G.M., Biurrun, F., Zerba, E., Picollo, M.I., 2006a. Fumigant and repellent properties of essential oils and component compounds against permethrin-resistant Pediculus humanus capitis (Anoplura: Pediculidae) from Argentina. J. Med. Entomol. 43, 889-895.

Toloza, A.C., Zygadlo, J., Mougabure-Cueto, G., Zerba, E., Faillaci, S., Picollo, M.I., 2006b. The fumigant and repellent activity of aliphatic lactones against Pediculus humanus capitis (Anoplura: Pediculidae). Mem. Inst. Oswaldo Cruz 101, 55-56.

Touhara, K., Vosshall, L.B., 2009. Sensing odorants and pheromones with chemosensory receptors. Annu. Rev. Physiol. 71, 307-332.

Veracx, A., Raoult, D., 2012. Biology and genetics of human head and body lice. Trends Parasitol. 28, 563-571.

Wang, G., Carey, A.F., Carlson, J.R., Zwiebel, L.J., 2010. Molecular basis of odor coding in the malaria vector mosquito Anopheles gambiae. Proc. Natl. Acad. Sci. U. S. A. 107, 4418-4423.

Xu, P., Choo, Y.M., Pelletier, J., Sujimoto, F.R., Hughes, D.T., Zhu, F., Atungulu, E., Cornel, A.J., Luetje, C.W., Leal, W.S., 2013. Silent, generic and plant kairomone sensitive odorant receptors from the southern house mosquito. J. Insect Physiol. 59, 961-966.

Yoon, K.S., Strycharz, J.P., Gao, J.R., Takano-Lee, M., Edman, J.D., Clark, J.M., 2006. An improved in vitro rearing system for the human head louse allows the determination of resistance to formulated pediculicides. Pestic. Biochem. Phys. 86, 195-202. 